\title{
Effect of Dexmedetomidine on Postoperative Sleep Quality: A Systematic Review
}

\author{
Xiao Huang (iD* \\ Dandan Lin* \\ Yi Sun \\ Anshi Wu \\ Changwei Wei (ID)
}

Department of Anesthesiology, Beijing Chao-Yang Hospital, Capital Medical University, Beijing, People's Republic of China

*These authors contributed equally to this work
Correspondence: Changwei Wei Department of Anesthesiology, Beijing Chao-Yang Hospital, Capital Medical

University, No. 8, South Workers Stadium Road, Chaoyang District, Beijing, People's Republic of China

Tel +86 I0-85231330

Email changwei.wei@ccmu.edu.cn

\begin{abstract}
In this article, we conduct a systematic review of the literature to explore the specific role of dexmedetomidine (DEX) on postoperative sleep and its associated mechanisms at present. The electronic database Embase, MEDLINE/PubMed, the Cochrane Library, Web of Science, and Google Scholar were searched. The restriction terms included "dexmedetomidine", "sleep" and "surgery". The inclusion criteria were as following: 1) patients 18 years old or older; 2) DEX used in the perioperative period not just for critically ill patients in the intensive care unit (ICU); 3) prospective or retrospective studies. The review articles, conference abstracts, and animal studies were excluded. Out of the 22 articles which met the above criteria, 20 of them were randomized controlled studies and 2 of them were retrospective cohort studies. Infusion of DEX including during the surgery and after surgery at a low or high dose was shown to improve subjective and objective sleep quality, although 2 studies showed there is no evidence that the use of DEX improves sleep quality and 1 showed less sleep efficiency and shorter total sleep time in the DEX group. Other postoperative outcomes evaluated postoperative nausea and vomiting, pain, postoperative delirium bradycardia and hypotension. Outcomes of our systematic review showed that DEX has advantages in improving patients' postoperative sleep quality. Combined with the use of general anesthetic, DEX provides a reliable choice for procedural sedation.
\end{abstract}

Keywords: dexmedetomidine, sleep, surgery

\section{Introduction}

Patients often suffer from severe sleep disturbances after surgery. The manifestations of polysomnography usually include severe sleep deprivation, sleep fragmentation, reduced duration of slow-wave sleep, and rapid eye movement sleep. Patients may present with reduced sleep duration, increased awakenings, reduced sleep quality, and frequent nightmares. ${ }^{1}$ Clinical and experimental studies suggest that sleep disturbances may exacerbate pain. Surgical stress appears to be the main cause of sleep disruption. Disturbed sleep and sleep-wake rhythms are serious problems in postoperative patients and may lead to prolonged postoperative recovery time, ${ }^{2}$ increased incidence of cardiovascular disease, cognitive dysfunction, ${ }^{3}$ and impaired immune function. ${ }^{4}$ Increased morbidity and mortality are associated with more pronounced rest-activity rhythms.

Dexmedetomidine (DEX) was first approved by the FDA in 1999 for sedation of intensive care patients. DEX is a highly selective $\alpha-2$ adrenergic agonist. DEX can improve sedation and analgesia, reduces the incidence of postoperative delirium, and improves sleep quality. ${ }^{5,6}$ However, Tan et al reported that patients undergoing transurethral resection of the prostate (TURP) had poorer sleep quality at night after 
spinal anesthesia due to the deeper state of sedation provided by DEX during the daytime. ${ }^{7}$ Therefore, the effects of intraoperative DEX use at different time periods on postoperative sleep quality need to be further investigated.

Little is known about the specific role of DEX on postoperative sleep and its associated mechanisms at present. Hence it is important to study the circadian rhythm changes of perioperative DEX on postoperative body function and sleep and to understand the postoperative pathophysiological processes. A better understanding of patients with postoperative sleep disorders and the search for effective prevention and treatment may facilitate the development of more effective and safer sleep management programs. This review will focus on the basic science and clinical evidence to determine its efficacy in improving postoperative sleep.

\section{Methods}

We searched the electronic database Embase, MEDLINE/ PubMed, the Cochrane Library, Web of Science, and Google Scholar. The search started in September 2020 and was completed in November 2020 without language limitation. The following search terms were used to form the basic search strategy: "dexmedetomidine", "sleep" and "surgery".

The title and abstract of records were screened by 2 reviewers independently. And the included records should report the sleep quality or sleep pattern in the perioperative period. Then full-text studies were measured for eligibility by inclusion criteria as follows: (1) patients 18 years old or older; (2) DEX used in the perioperative period not just for critically ill patients in the intensive care unit (ICU); (3) prospective or retrospective studies. The review articles, conference abstracts, and animal studies were excluded. The level of evidence for each study was classified by 2 reviewers according to the published guidelines.

\section{Results}

\section{Identification of Studies}

The flow chart of the study is shown in Figure 1. Out of the 22 studies evaluated, 20 were randomized controlled trials (RCTs), and 2 of them were retrospective studies. All studies are about non-cardiac surgery except a study by

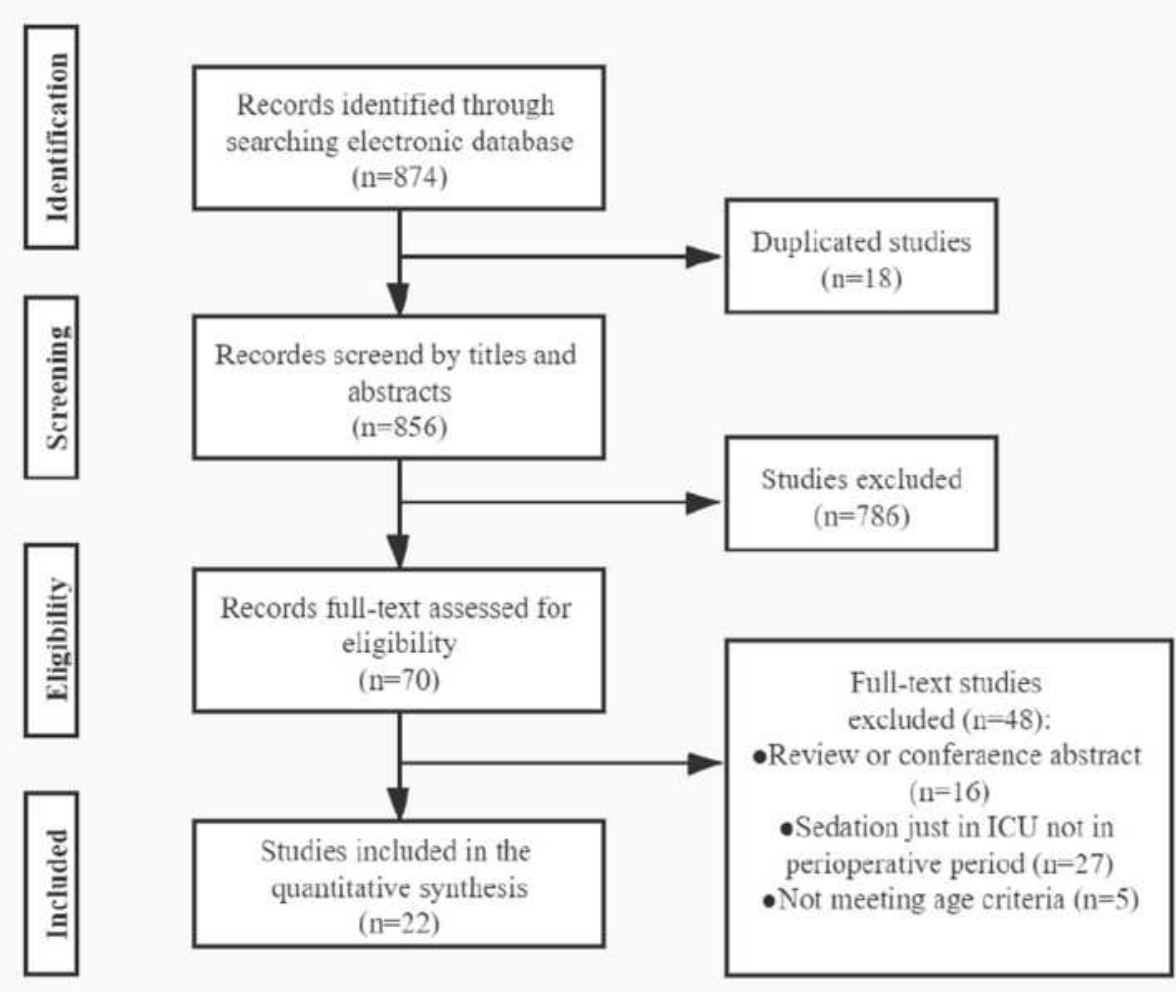

Figure I Flow chart of the study. Abbreviation: ICU, intensive care unit. 
Corbett et al. ${ }^{8}$ Seventeen of them compared DEX to placebo, one study compared DEX plus sufentanil to sufentanil anesthesia, and one study compared DEX plus postfascia iliaca compartment block (FCIB) to FCIB. The mean age of patients included in the study ranged from 31 to 76 . The evaluated articles are described in Table 1.

\section{Sleep Quality}

\section{Objective Sleep Evaluation}

Nineteen studies used objective tools to evaluate perioperative sleep quality. Different questionnaires were used in the studies according to the choice of authors. The Numeric Rating Scales (NRS) for sleep quality was the most used questionnaire in nine studies, ${ }^{9-17}$ followed by Pittsburgh Sleep Quality Index (PSQI) in two studies, ${ }^{7,18}$ Richard Campbell Sleep Questionnaire (RCSQ) in one study, ${ }^{19}$ St. Mary Hospital Sleep Questionnaire in one study, ${ }^{20}$ the insomnia severity index (ISI) in one study, ${ }^{21}$ Athens insomnia scale in one study, the Consensus Sleep Diary in one study $^{22}$ and other simple questions in three studies. ${ }^{8,23,24}$

\section{Subjective Sleep Evaluation}

Seven studies evaluated perioperative sleep quality through subjective tools. Polysomnography (PSG) was used in three studies. ${ }^{11,12,25}$ And other subjective tools included bispectral index (BIS) in three studies ${ }^{7,26,27}$ and portable sleep monitor in one study. ${ }^{5}$ PSG and portable sleep monitor could analyze multiple factors of sleep including, sleep efficiency index, arousal index, the percentage of REM, stage 1, 2, and 3. BIS could analyze the sleep efficiency index and total sleep time. The time of subjective evaluations was most in 1, 2, and 3 days after surgery. Objective and subjective evaluations of sleep quality of patients in the perioperative period were shown in Table 2.

\section{Usage and Dose of DEX}

As for the different time point, the DEX was used during the surgery for sedation in nine studies, ${ }^{5,7,10,15-18,21,26}$ as an adjuvant for the regional block in one studies, ${ }^{22}$ for postoperative analgesia in nine studies ${ }^{8,12-14,19,23-25,27}$ and both in surgery and after surgery in eight studies. ${ }^{9,11,20}$ And in 2 retrospective study ${ }^{15,16}$ and one $\mathrm{RCT}^{25}$ compared the effect of sleep quality of low dose of DEX $(<0.2 \mu \mathrm{g} /$ $\mathrm{kg} / \mathrm{h})$ and high dose $(>0.2 \mu \mathrm{g} / \mathrm{kg} / \mathrm{h})$. As for other RCT studies, nine studies used DEX at a low dose and ten studies used DEX at a high dose.

\section{Other Outcomes}

Postoperative Nausea and Vomiting (PONV)

Shi et al found that DEX reduced the incidence of postoperative vomiting but not the incidence of nausea. ${ }^{23}$ Another study by Li et al showed that DEX reduced the incidence of PONV. ${ }^{14}$ Five other studies found no significant benefit of DEX in reducing PONV. ${ }^{10,16,18,19,24}$

\section{Pain}

A total of 13 studies have compared the effects of DEX on postoperative pain. The pain was assessed by numeric rating scales, visual analog scales (VAS), or the use of the postoperative pain reliever. Of these studies, 8 studies found DEX could relieve postoperative pain. ${ }^{12-14,17,18,21,22,25}$ Four studies concluded DEX does not effectively relieve pain after surgery. ${ }^{9,15,16,20}$ A study by Corbett et al reported that ICU patients sedated with DEX exhibited more severe pain compared with propofol. ${ }^{8}$

\section{Postoperative Delirium}

$\mathrm{Su}$ et al conducted a study on patients over 65 years admitted to the ICU after non-cardiac surgery and found that prophylactic low-dose DEX significantly reduced the incidence of delirium within 7 days after surgery. ${ }^{13}$ However, 3 other studies suggested that perioperative infusion of DEX did not reduce the incidence of postoperative delirium after surgery. ${ }^{14,19,20}$ A study by Shi showed that compared with the placebo group, continuous infusion of DEX $(0.5 \mathrm{~g} / \mathrm{kg} / \mathrm{h})$ in patients undergoing thoracoscopic lobectomy reduced the incidence of postoperative cognitive dysfunction at 7 days postoperatively, but did not reduce the incidence of postoperative delirium. ${ }^{17}$

\section{Cardiovascular Events}

We selected postoperative bradycardia and hypotension as primary adverse events with DEX. Twelve studies compared perioperative blood pressure changes between the treatment group and the control group. And 6 of these studies showed that perioperative use of DEX increased the incidence of hypotension or significantly lowered blood pressure compared to the controls group. ${ }^{11-13,15,24,25}$ The other 6 studies did not find associations between the use of DEX and hypotension. ${ }^{14,17,19,23,26,27}$ Nine studies compared the effect of perioperative DEX versus the control group on postoperative bradycardia. Five studies demonstrated that the use of DEX increased the incidence of postoperative bradycardia or significantly reduced heart rate compared to the control group, ${ }^{12,15,17,24,27}$ and 4 studies did not show that postoperative bradycardia is associated with DEX. ${ }^{14,19,25,26}$ 
Table I Studies Included in This Systematic Review

\begin{tabular}{|c|c|c|c|c|c|c|c|}
\hline & $\begin{array}{l}\text { Type of } \\
\text { Study }\end{array}$ & $\begin{array}{l}\text { Sample } \\
\text { Size }\end{array}$ & $\begin{array}{l}\text { Age } \\
\text { (Con/ } \\
\text { Dex) }\end{array}$ & Surgery & Interventions & Control & $\begin{array}{l}\text { Level of } \\
\text { Evidence }\end{array}$ \\
\hline $\begin{array}{l}\text { Mao et al, } \\
2020^{20}\end{array}$ & $\mathrm{RCT}$ & 56 & $63 / 65$ & $\begin{array}{l}\text { Lateral } \\
\text { thoracotomy }\end{array}$ & $\begin{array}{l}10 \text { mins befor induction: DEX } 0.5 \\
\mu \mathrm{g} / \mathrm{kg} / \mathrm{h} \text {; During surgery: DEX } \\
0.2-0.4 \mu \mathrm{g} / \mathrm{kg} / \mathrm{h} \text {; For } 5 \text { days after } \\
\text { surgery: DEX } 0.06 \mu \mathrm{g} / \mathrm{kg} / \mathrm{h}\end{array}$ & Placebo & 1 \\
\hline $\begin{array}{l}\text { Liu et al, } \\
2020^{18}\end{array}$ & $\mathrm{RCT}$ & 119 & $\begin{array}{l}70 / 70 / \\
70\end{array}$ & $\begin{array}{l}\text { Total hip } \\
\text { arthroplasty }\end{array}$ & $\begin{array}{l}\text { During surgery: DEX } 0.6 \mu \mathrm{g} / \mathrm{kg} \text {; } \\
\text { After surgery: } \mathrm{FCIB}\end{array}$ & Placebo; FCIB & II \\
\hline $\begin{array}{l}\text { Shi et al, } \\
2020^{17}\end{array}$ & $\mathrm{RCT}$ & 106 & $69 / 69$ & $\begin{array}{l}\text { Thoracoscopic } \\
\text { lobectomy }\end{array}$ & During surgery: DEX $0.5 \mu \mathrm{g} / \mathrm{kg} / \mathrm{h}$ & Placebo & 1 \\
\hline $\begin{array}{l}\text { Duan et al, } \\
2020^{16}\end{array}$ & $\begin{array}{l}\text { Retrospective } \\
\text { cohort study }\end{array}$ & 7418 & $54 / 53$ & $\begin{array}{l}\text { Non-cardiac } \\
\text { surgery }\end{array}$ & During surgery: DEX $0.2-0.7 \mu \mathrm{g} / \mathrm{kg} / \mathrm{h}$ & Placebo & III \\
\hline $\begin{array}{l}\text { Cai et al, } \\
2020^{15}\end{array}$ & $\begin{array}{l}\text { Retrospective } \\
\text { cohort study }\end{array}$ & 4379 & $\begin{array}{l}72 / 71 / \\
71\end{array}$ & $\begin{array}{l}\text { Non-cardiac } \\
\text { surgery }\end{array}$ & $\begin{array}{l}\text { During surgery low dose: DEX } \\
0 . \mathrm{I}-0.2 \mu \mathrm{g} / \mathrm{kg} / \mathrm{h} \text {; During surgery high } \\
\text { dose: } \mathrm{DEX}>0.2 \mu \mathrm{g} / \mathrm{kg} / \mathrm{h}\end{array}$ & Placebo & III \\
\hline $\begin{array}{l}\text { Song et al, } \\
2019^{5}\end{array}$ & $\mathrm{RCT}$ & 75 & $42 / 43$ & $\begin{array}{l}\text { Laproscopic } \\
\text { abdominal } \\
\text { surgery }\end{array}$ & $\begin{array}{l}\text { During surgery in day group and } \\
\text { night group }\end{array}$ & None & II \\
\hline $\begin{array}{l}\text { Sun et al, } \\
2019^{19}\end{array}$ & $\mathrm{RCT}$ & 557 & $68 / 69$ & $\begin{array}{l}\text { Non-cardiac } \\
\text { surgery }\end{array}$ & $\begin{array}{l}\text { PCIA: DEX } 0.1 \mu g / k g / h \text { with } \\
\text { sufentanil } 2 \mu g / \mathrm{kg} \text { and tropisetron } \\
6 \mathrm{mg}\end{array}$ & $\begin{array}{l}\text { PCIA: } 2 \mu \mathrm{g} / \mathrm{kg} \\
\text { sufentanil and } 6 \mathrm{mg} \\
\text { tropisetron }\end{array}$ & 1 \\
\hline $\begin{array}{l}\text { Yu et al, } \\
2019^{21}\end{array}$ & $\mathrm{RCT}$ & 557 & $3|/ 3|$ & $\begin{array}{l}\text { Cesarean } \\
\text { surgery }\end{array}$ & $\begin{array}{l}\text { During surgery after delivery: DEX } \\
0.5 \mu \mathrm{g} / \mathrm{kg} \text { for } 20 \mathrm{mins} \text {; PCIA: DEX } \\
150 \mu \mathrm{g} \text { and sufentanil I } 50 \mu \mathrm{g}\end{array}$ & $\begin{array}{l}\text { Placebo after } \\
\text { delivery; } \\
\text { PCIA: sufentanil } \\
\text { I50 } \mu \mathrm{g}\end{array}$ & 1 \\
\hline $\begin{array}{l}\text { Li et al, } \\
2018^{14}\end{array}$ & $\begin{array}{l}\text { RCT pilot } \\
\text { study }\end{array}$ & 58 & $67 / 69$ & $\begin{array}{l}\text { Open } \\
\text { abdominal } \\
\text { surgery }\end{array}$ & $\begin{array}{l}\text { PCIA: DEX } 2 \mu g / \mathrm{mL} \text { and } 0.5 \mathrm{mg} / \mathrm{mL} \\
\text { morphine }\end{array}$ & $\begin{array}{l}\text { PCIA: } 0.5 \mathrm{mg} / \mathrm{mL} \\
\text { morphine }\end{array}$ & II \\
\hline Author & Type of study & $\begin{array}{l}\text { Sample } \\
\text { size }\end{array}$ & $\begin{array}{l}\text { Age } \\
\text { (Con/ } \\
\text { Dex) }\end{array}$ & Surgery & Interventions & Control & $\begin{array}{l}\text { Level of } \\
\text { evidence }\end{array}$ \\
\hline $\begin{array}{l}\text { Su et al, } \\
2017^{13}\end{array}$ & $\mathrm{RCT}$ & 700 & - & $\begin{array}{l}\text { Non-cardiac } \\
\text { surgery }\end{array}$ & $\begin{array}{l}\text { After surgery: } 0.1 \mu g / \mathrm{kg} / \mathrm{h} \text { to the } \\
0800 \mathrm{~h} \text { on postoperative day I }\end{array}$ & Placebo & I \\
\hline $\begin{array}{l}\text { Chen et al, } \\
2017^{12}\end{array}$ & $\mathrm{RCT}$ & 60 & $45 / 43$ & $\begin{array}{l}\text { Abdominal } \\
\text { hysterectomy }\end{array}$ & $\begin{array}{l}\text { PCIA: DEX } 0.05 \mu \mathrm{g} / \mathrm{kg} / \mathrm{h} \text { with } \\
\text { sufentanil } 0.02 \mu \mathrm{g} / \mathrm{kg} / \mathrm{h}\end{array}$ & $\begin{array}{l}\text { PCIA: sufentanil } \\
0.02 \mu \mathrm{g} / \mathrm{kg} / \mathrm{h}\end{array}$ & I \\
\hline $\begin{array}{l}\text { Qin et al, } \\
2017^{24}\end{array}$ & $\mathrm{RCT}$ & 60 & $58 / 59$ & $\begin{array}{l}\text { Partial } \\
\text { laryngectomy }\end{array}$ & $\begin{array}{l}\text { PCIA: DEX } 4 \mu g / m L \text { with sufentanil } \\
\text { I } \mu g / m L\end{array}$ & $\begin{array}{l}\text { PCIA: sufentanil I } \\
\mu \mathrm{g} / \mathrm{mL}\end{array}$ & II \\
\hline $\begin{array}{l}\text { Lu et al, } \\
2017^{27}\end{array}$ & Cohort study & 20 & $52 / 45$ & $\begin{array}{l}\text { Abdominal } \\
\text { surgery }\end{array}$ & $\begin{array}{l}\text { After surgery: } 0.2-0.7 \mu \mathrm{g} / \mathrm{kg} / \mathrm{h} \\
\text { during first night after surgery } \\
(20: 00-8: 00)\end{array}$ & Placebo & II \\
\hline $\begin{array}{l}\text { Shi et al, } \\
2017^{23}\end{array}$ & $\mathrm{RCT}$ & 46 & $48 / 49$ & $\begin{array}{l}\text { Radical } \\
\text { mastectomy }\end{array}$ & $\begin{array}{l}\text { I5 mins before surgery: I } \mu g / \mathrm{kg} \text {; } \\
\text { During surgery: } 0.4 \mu \mathrm{g} / \mathrm{kg} / \mathrm{h}\end{array}$ & Placebo & II \\
\hline
\end{tabular}

(Continued) 
Table I (Continued).

\begin{tabular}{|c|c|c|c|c|c|c|c|}
\hline & $\begin{array}{l}\text { Type of } \\
\text { Study }\end{array}$ & $\begin{array}{l}\text { Sample } \\
\text { Size }\end{array}$ & $\begin{array}{l}\text { Age } \\
\text { (Con/ } \\
\text { Dex) }\end{array}$ & Surgery & Interventions & Control & $\begin{array}{l}\text { Level of } \\
\text { Evidence }\end{array}$ \\
\hline $\begin{array}{l}\text { Wu et al, } \\
2016^{11}\end{array}$ & $\begin{array}{l}\text { RCT pilot } \\
\text { study }\end{array}$ & 76 & $76 / 74$ & $\begin{array}{l}\text { Non-cardiac } \\
\text { surgery }\end{array}$ & $\begin{array}{l}\text { DEX } 0.1 \mu \mathrm{g} / \mathrm{kg} / \mathrm{h} \text { from } 5: 00 \mathrm{PM} \text { on } \\
\text { the day of surgery to } 8: 00 \mathrm{AM} \text { on } \\
\text { the Ist day after surgery }\end{array}$ & Placebo & I \\
\hline $\begin{array}{l}\text { Jiang et al, } \\
2018^{25}\end{array}$ & RCT & 99 & $\begin{array}{l}65 / 64 / \\
64\end{array}$ & $\begin{array}{l}\text { Abdominal } \\
\text { surgery }\end{array}$ & $\begin{array}{l}\text { PCIA DI: DEX } 2.4 \mu g / k g \text { with } \\
\text { oxycodone } 0.6 \mathrm{mg} / \mathrm{kg} \text {; PCIA D2: } \\
\text { DEX } 4.8 \mu \mathrm{gg} / \mathrm{kg} \text { with oxycodone } \\
0.6 \mathrm{mg} / \mathrm{kg}\end{array}$ & $\begin{array}{l}\text { PCIA: oxycodone } \\
0.6 \mathrm{mg} / \mathrm{kg}\end{array}$ & II \\
\hline $\begin{array}{l}\text { Tan et al, } \\
2016^{7}\end{array}$ & $\mathrm{RCT}$ & 66 & $\begin{array}{l}71 / 70 / \\
73\end{array}$ & TURP & During surgery: DEX $1.2 \mu \mathrm{g} / \mathrm{kg} / \mathrm{h}$ & $\begin{array}{l}\text { Placebo or } \\
\text { midazolam } \\
0.225 \mathrm{mg} / \mathrm{kg} / \mathrm{h}\end{array}$ & I \\
\hline $\begin{array}{l}\text { Tan et al, } \\
2016^{26}\end{array}$ & $\mathrm{RCT}$ & 161 & $\begin{array}{l}56 / 53 / \\
56\end{array}$ & $\begin{array}{l}\text { Thoracic } \\
\text { surgery }\end{array}$ & During surgery: DEX $\mid \mu g / \mathrm{kg} / \mathrm{h}$ & Placebo & ॥ \\
\hline $\begin{array}{l}\text { Chen et al, } \\
2016^{10}\end{array}$ & $\mathrm{RCT}$ & 60 & $60 / 57$ & $\begin{array}{l}\text { Laparoscopic } \\
\text { colorectal } \\
\text { resection }\end{array}$ & $\begin{array}{l}10 \text { mins before surgery: DEX I } \mu \mathrm{g} / \\
\mathrm{kg} \text {; During surgery: DEX } 0.3 \mu \mathrm{g} / \mathrm{kg} / \mathrm{h}\end{array}$ & Placebo & 1 \\
\hline Author & Type of study & $\begin{array}{l}\text { Sample } \\
\text { size }\end{array}$ & $\begin{array}{l}\text { Age } \\
\text { (Con/ } \\
\text { Dex) }\end{array}$ & Surgery & Interventions & Control & $\begin{array}{l}\text { Level of } \\
\text { evidence }\end{array}$ \\
\hline $\begin{array}{l}\text { Ghali et al, } \\
2015^{22}\end{array}$ & RCT & 60 & $62 / 61$ & $\begin{array}{l}\text { Vitreoretinal } \\
\text { surgery }\end{array}$ & $\begin{array}{l}\text { Regional anesthesia: DEX } 20 \mu \mathrm{g} \text { with } \\
0.75 \% \text { levobupivacaine } 4 \mathrm{~mL} \text { and } \mathrm{I} 5 \\
\text { IU hyaluronidase }\end{array}$ & $\begin{array}{l}\text { Regional anesthesia: } \\
0.75 \% \\
\text { levobupivacaine } \\
4 \mathrm{~mL} \text { and } 15 \mathrm{IU} \\
\text { hyaluronidase }\end{array}$ & 1 \\
\hline $\begin{array}{l}\text { Yang et al, } \\
2015^{9}\end{array}$ & $\mathrm{RCT}$ & 79 & $51 / 50$ & $\begin{array}{l}\text { Microvascular } \\
\text { free flap } \\
\text { surgery }\end{array}$ & $\begin{array}{l}\text { During surgery: DEX } 0.5 \mu \mathrm{g} / \mathrm{kg} / \mathrm{h} \text {; } \\
\text { After surgery: DEX } 0.2-0.7 \mu \mathrm{g} / \mathrm{kg} / \mathrm{h} \\
\text { to the } 6: 00 \text { AM next morning }\end{array}$ & Placebo & 1 \\
\hline $\begin{array}{l}\text { Corbett } \\
\text { et al, } 2005^{8}\end{array}$ & $\mathrm{RCT}$ & 89 & $62 / 64$ & CABG & After surgery: DEX $0.4 \mu \mathrm{g} / \mathrm{kg} / \mathrm{h}$ & $\begin{array}{l}\text { After surgery: } \\
\text { propofol } 0.2-0.7 \\
\mu g / \mathrm{kg} / \mathrm{h}\end{array}$ & ॥ \\
\hline
\end{tabular}

Abbreviations: RCT, randomized controlled trials; DEX, dexmedetomidine; FCIB, post-fascia iliaca compartment block; PCIA, patient-controlled intravenous analgesia; TURP, transurethral resection of the prostate; CABG, coronary artery bypass grafting.

\section{Others}

Sun et al concluded that continuous postoperative infusion of DEX reduced the incidence of postoperative pneumonia in elderly patients undergoing general surgery. ${ }^{19}$ Qin et al reported that the combination of DEX/sufentanil in patients undergoing partial laryngectomy significantly reduced the dosage of sufentanil and the frequency of postoperative coughing episodes. ${ }^{24}$ Shi et al suggested that DEX could reduce postoperative anxiety, ${ }^{17}$ and another study by Shi et al also found that postoperative fatigue severity scores were significantly lower in the DEX group than in the control group. ${ }^{23}$
Whereas Corbett et al did not find the effectiveness of DEX in relieving postoperative anxiety. ${ }^{8}$ Other perioperative outcomes of the use of DEX in the perioperative period were shown in Table 3.

\section{Discussion}

In this review, our findings suggest that intraoperative or postoperative infusion of DEX may be adequate to improve sleep quality within a short time period after surgery. Although there were many variables that measure the quality of sleep, we find the preventive effect of DEX 
Table 2 Objective and Subjective Evaluations of Sleep Quality of Patients in Perioperative Period

\begin{tabular}{|c|c|c|c|}
\hline Evaluation & Evaluation Content & Result & $\begin{array}{l}\text { Supporting } \\
\text { Studies }\end{array}$ \\
\hline \multicolumn{4}{|c|}{ Subjective tools } \\
\hline SMH & $\begin{array}{l}\text { Subjective sleep quality, clear-headed after getting up in the morning, } \\
\text { sleep satisfaction }\end{array}$ & $\begin{array}{l}\text { DEX: improved sleep quality on POD2 and POD5; more clear- } \\
\text { headed after getting up on POD5; no differences in sleep } \\
\text { satisfaction }\end{array}$ & $\begin{array}{l}\text { During and } \\
\text { after surgery }\end{array}$ \\
\hline PSQI & $\begin{array}{l}\text { Subjective sleep quality, sleep latency, sleep duration, habitual sleep } \\
\text { efficiency, sleep disturbances, use of sleeping medication, and } \\
\text { daytime dysfunction }\end{array}$ & DEX: lower PSQI scores, improved subjective sleep quality & $\begin{array}{l}\text { During } \\
\text { surgery }\end{array}$ \\
\hline NRS & Subjective sleep quality, a scale of $0-10$ & $\begin{array}{l}\text { DEX: improved subjective sleep quality; better sleep quality in } \\
\text { low dose group; lower incidence of sleep disturbance } \\
\text { DEX: }\end{array}$ & $\begin{array}{l}\text { During } \\
\text { surgery } \\
\text { After surgery } \\
\text { During and } \\
\text { after surgery }\end{array}$ \\
\hline RCSQ & $\begin{array}{l}\text { Wake after sleep onset, sleep depth, sleep quality, the severity of } \\
\text { sleep-onset, the severity of going back to sleep if awakened }\end{array}$ & DEX: higher subjective quality on POD I, 2 and 3 & After surgery \\
\hline$|S|$ & $\begin{array}{l}\text { The severity of sleep-onset, sleep maintenance difficulties, } \\
\text { satisfaction with current sleep pattern, interference with daily } \\
\text { functioning, noticeability impairment and degree of distress or } \\
\text { concern caused by sleep disorder }\end{array}$ & DEX: lower sleep score on POD2 not in POD7 or POD42 & $\begin{array}{l}\text { During } \\
\text { surgery }\end{array}$ \\
\hline AIS & $\begin{array}{l}\text { Sleep latency, awakenings during the night, final awakening earlier } \\
\text { than desired, sleep duration, sleep quality, sense of well-being } \\
\text { functioning (physical and mental) and sleepiness during the day }\end{array}$ & $\begin{array}{l}\text { DEX: improved subjective sleep quality on POD } 2 \text { and } 3 \text { not on } \\
\text { PODI in day group }\end{array}$ & $\begin{array}{l}\text { During and } \\
\text { after surgery }\end{array}$ \\
\hline CSD & The quality of sleep (very poor, poor, fair, good, very good) & DEX: higher rates of good sleep quality on PODI & $\begin{array}{l}\text { During } \\
\text { surgery }\end{array}$ \\
\hline $\begin{array}{l}\text { Other } \\
\text { simple } \\
\text { questions }\end{array}$ & Total sleep time; the quality of sleep & DEX: longer total sleep time on PODI & $\begin{array}{l}\text { During } \\
\text { surgery }\end{array}$ \\
\hline Evaluation & Evaluation content & Result & $\begin{array}{l}\text { Supporting } \\
\text { studies }\end{array}$ \\
\hline & & DEX: more sleeping difficulty; less sleeping disturbance & After surgery \\
\hline \multicolumn{4}{|c|}{ Objective tools } \\
\hline $\begin{array}{l}\text { Portable } \\
\text { sleep } \\
\text { monitor }\end{array}$ & $\begin{array}{l}\text { Sleep efficiency, the percentage of REM sleep, unstable and stable } \\
\text { sleep }\end{array}$ & $\begin{array}{l}\text { DEX: higher sleep efficiency, longer unstable sleep in day } \\
\text { group; lower REM sleep and stable sleep in night group }\end{array}$ & $\begin{array}{l}\text { During and } \\
\text { after surgery }\end{array}$ \\
\hline PSG & $\begin{array}{l}\text { The sleep efficiency index, arousal index and percentage of REM, and } \\
\text { stage I, 2, and } 3 \text { sleep }\end{array}$ & $\begin{array}{l}\text { DEX: improved sleep efficiency index, lower arousal index, } \\
\text { higher N2 sleep, lower NI sleep and no differences in REM } \\
\text { and N3 sleep on POD I and } 2\end{array}$ & After surgery \\
\hline BIS & $\begin{array}{l}\text { The level of sedation, sleep depth, sleep efficiency index and total } \\
\text { sleep time }\end{array}$ & $\begin{array}{l}\text { DEX: higher sleep efficiency and longer total sleep time; } \\
\text { DEX: less sleep efficiency and shorter total sleep time; no } \\
\text { differences in sleep efficiency on POD I and } 2\end{array}$ & $\begin{array}{l}\text { After surgery } \\
\text { During } \\
\text { surgery }\end{array}$ \\
\hline
\end{tabular}

Abbreviations: SMH, St. Mary Hospital Sleep Questionnaire; PSQI, Pittsburgh Sleep Quality Index; NRS, Numeric Rating Scales; RCSQ, Richards Campbell Sleep Questionnaire; ISI, insomnia severity index; AIS, Athens Insomnia Scale; CSD, the consensus sleep diary; PSG, polysomnography; BIS, bispectral index. 
Table 3 Other Perioperative Outcomes of Use of Dexmedetomidine in Perioperative Period

\begin{tabular}{|l|}
\hline Other Perioperative Outcomes \\
\hline Postoperative nausea and vomiting \\
\hline Pain \\
\hline Perioperative delirium \\
\hline $\begin{array}{l}\text { Adverse events } \\
\text { Bradycardia } \\
\text { Hypotension }\end{array}$ \\
\hline
\end{tabular}

to reduce sleep disturbance caused by surgery and anesthesia. Additionally, infusion of DEX in the perioperative period could release postoperative pain, reduce the incidence of postoperative delirium, prevent PONV and so on.

In a real-world cohort study of 7418 patients, infusion of DEX reduced the incidence of postoperative sleep disturbance. ${ }^{16}$ One of the potential mechanisms of improving the sleep quality on the first or second night after surgery is the changing sleep structure by DEX. The sleep cycle could be divided into non-rapid eye movement sleep (NREM) and rapid eye movement sleep (REM). And NREM could be further divided into stages N1, N2 and $\mathrm{N} 3{ }^{28}$

In animal experiments, it was found that transoral administration of DEX decreased c-Fos expression in the cerebral cortex and subcortical arousal system and increased c-Fos expression in ventral lateral preoptic nucleus neurons in mice. DEX may promote NREM sleep in mice by promoting excitation in the sleep nucleus and inhibiting the arousal nucleus. ${ }^{29}$ Nelson et al suggested that endogenous sleep pathways involved in DEX induced sedation which inhibited and disinhibited the locus ceruleus, disinhibited the ventral lateral preoptic nucleus firing. This increased the release of $\gamma$-aminobutyric acid receptor type A (GABA) from the terminal ventral lateral preoptic nucleus and inhibited tuberomammillary nucleus firing. ${ }^{30}$ The effect of $\alpha-2$ agonist DEX on NREM sleep may be mediated through postsynaptic $\alpha-2$ adrenoceptors. ${ }^{31}$ Some common changes in highfrequency oscillations could be detected in the thalamus and neocortex during DEX anesthesia. ${ }^{32}$

Surgery and anesthesia had a negative effect on sleep quality by changing the sleep pattern. REM and N3 sleep were significantly reduced in patients undergoing surgery.
At the same time, the N1 stage was longer for patients in the surgery group. ${ }^{12}$ Some studies indicated that continuous infusion of DEX could produce spindle and slow-delta oscillations, which represents the characteristic EEG features of stage 2 sleep. ${ }^{33-35}$ In healthy volunteers, oral DEX could improve stage 2 sleep and have a negative impact on REM sleep. ${ }^{36}$ This study provided evidence that DEX could modulate NREM sleep circuitry. Infusion DEX could also increase the $\mathrm{N} 3$ sleep in a dose-dependent manner, which is associated with improved cognition and synaptic plasticity. ${ }^{37,38}$ This suggested biomimetic N3 sleep may beneficial to cognition improvement.

Regarding intervention to mimic natural sleep and improve postoperative sleep quality, there is strong evidence that the use of DEX during and after surgery could improve the sleep quality assessed by objective and subjective measurements. Chen et al ${ }^{12}$ found infusion DEX improved the percentage of N2 sleep, reduced N1 sleep and reduced the arousal index. However, some researchers reported no differences or less sleep efficiency and shorter sleep time measured by BIS in DEX group. ${ }^{7,26}$

Timing of administration is associated with the effect of DEX on postoperative sleep quality. Song et $\mathrm{al}^{5}$ performed a prospective RCT to compare the effect of DEX in the daytime (8:00-12:00) and nighttime (18:00-22:00) surgery on sleep quality. They found that patients in the night group had shorter REM sleep and Stable sleep than those in the day group. This result indicated that infusion DEX in the daytime might have better improvement in sleep quality. Besides, the dose of DEX also had a different effect on postoperative sleep quality. Jiang et $\mathrm{al}^{25}$ examined the effect of the low and high dose of DEX combined with oxycodone on postoperative sleep quality. And Duan et $\mathrm{al}^{16}$ found that low dose DEX may be an optimal treatment of postoperative sleep disturbance.

DEX and other postoperative patient outcomes are controversial at present. A systematic review study concluded that DEX resulted in a reduction in pain compared with midazolam sedation and that both had similar respiratory and hemodynamic safety. ${ }^{39}$ Some studies suggest that DEX can cause hypotension or bradycardia, however, there is controversy in this field. The different modes of administration and doses of DEX may be also subject to safety issues, and patients with perioperative use of DEX should be closely monitored. A meta-analysis by Constantin et $\mathrm{al}^{40}$ found that DEX reduced the length of ICU stay and duration of mechanical ventilation and decreased the incidence of delirium, but also increased 
the incidence of bradycardia and hypotension. Although in our retrospective study we did not conclude that DEX reduced postoperative delirium or had significantly unsafe outcomes.

The recent study also has the following limitations. The included studies varied widely in the dosing regimen, the timing of administration, patient population, outcome, and follow-up. Moreover, not all studies reported on these outcome measures have sufficient detail. This stopped us from doing more formal meta-analyses. The studies we included ranged from low to high quality. And we included patients on DEX in the ICU, which increases the heterogeneity of the article. We note that most of the studies we included were in non-cardiac surgery. There is no definite conclusion about the effect of perioperative DEX on postoperative sleep and its safety in patients undergoing cardiac surgery at present. Our studies included were all perioperative DEX assisted sedation for general anesthesia surgery, while the effectiveness of DEX use in intravertebral anesthesia is unknown. Zhang et al concluded that DEX was generally safe and well-tolerated an additional agent to epidural surgery and improved sedation and analgesia. ${ }^{41}$ Studies of DEX-assisted nerve blocks have shown that $0.5-2 \mathrm{~g} / \mathrm{kg}$ DEX combined with $0.3 \%$ ropivacaine applied with TAP block is a safe and effective analgesic treatment in gynecological surgery. ${ }^{42}$

\section{Conclusion}

Outcomes of our systematic review showed that DEX has advantages in improving patients' postoperative sleep quality. Combined with the use of general anesthetic, DEX provides a reliable choice for procedural sedation.

\section{Abbreviations}

DEX, dexmedetomidine; TURP, transurethral resection of the prostate; ICU, intensive care unit; RCTs, randomized controlled trials; FCIB, post-fascia iliaca compartment block; NRS, Numeric Rating Scales; PSQI, Pittsburgh Sleep Quality Index; ISI, insomnia severity index; PSG, Polysomnography; BIS, bispectral index; PONV, Postoperative nausea and vomiting; VAS, visual analog scales; NREM, non-rapid eye movement sleep; REM, rapid eye movement sleep; GABA, $\gamma$-aminobutyric acid receptor type $\mathrm{A}$.

\section{Acknowledgment}

Xiao Huang and Dandan Lin share the first authorship.

\section{Funding}

There was no external funding.

\section{Disclosure}

The authors have no financial or other relationships relevant to this article.

\section{References}

1. Chouchou F, Khoury S, Chauny JM, et al. Postoperative sleep disruptions: a potential catalyst of acute pain? Sleep Med Rev. 2014;18 (3):273-282. doi:10.1016/j.smrv.2013.07.002

2. Gögenur I, Bisgaard T, Burgdorf S, et al. Disturbances in the circadian pattern of activity and sleep after laparoscopic versus open abdominal surgery. Surg Endosc. 2009;23(5):1026-1031. doi:10.1007/s00464-008-0112-9

3. Ni P, Dong H, Zhou Q, et al. Preoperative sleep disturbance exaggerates surgery-induced neuroinflammation and neuronal damage in aged mice. Mediators Inflamm. 2019;2019(2019):8301725. doi:10.1155/2019/8301725

4. Ruiz FS, Andersen ML, Guindalini C, et al. Sleep influences the immune response and the rejection process alters sleep pattern: evidence from a skin allograft model in mice. Brain Behav Immun. 2017;61:274-288. doi:10.1016/j.bbi.2016.12.027;.

5. Song B, Li Y, Teng X, et al. The effect of intraoperative use of dexmedetomidine during the daytime operation vs the nighttime operation on postoperative sleep quality and pain under general anesthesia. Nat Sci Sleep. 2019;11:207-215. doi:10.2147/nss. S225041;

6. Carr ZJ, Cios TJ, Potter KF, et al. Does dexmedetomidine ameliorate postoperative cognitive dysfunction? A brief review of the recent literature. Curr Neurol Neurosci Rep. 2018;18(10):64. doi:10.1007/ s11910-018-0873-z

7. Tan WF, Miao EY, Jin F, et al. Changes in first postoperative night bispectral index after daytime sedation induced by dexmedetomidine or midazolam under regional anesthesia: a Randomized Controlled Trial. Reg Anesth Pain Med. 2016;41(3):380-386. doi:10.1097/ aap. 0000000000000370

8. Corbett SM, Rebuck JA, Greene CM, et al. Dexmedetomidine does not improve patient satisfaction when compared with propofol during mechanical ventilation. Crit Care Med. 2005;33(5):940-945. doi:10.1097/01.ccm.0000162565.18193.e5

9. Yang X, Li Z, Gao C, et al. Effect of dexmedetomidine on preventing agitation and delirium after microvascular free flap surgery: a randomized, double-blind, control study. J Oral Maxillofac Surg. 2015;73(6):1065-1072. doi:10.1016/j.joms.2015.01.011

10. Chen C, Huang P, Lai L, et al. Dexmedetomidine improves gastrointestinal motility after laparoscopic resection of colorectal cancer: a randomized clinical trial. Medicine (Baltimore). 2016;95(29):e4295. doi:10.1097/md.0000000000004295

11. Wu XH, Cui F, Zhang C, et al. Low-dose dexmedetomidine improves sleep quality pattern in elderly patients after noncardiac surgery in the intensive care unit: a Pilot Randomized Controlled Trial. Anesthesiology. 2016;125(5):979-991. doi:10.1097/ aln.0000000000001325

12. Chen Z, Tang R, Zhang R, et al. Effects of dexmedetomidine administered for postoperative analgesia on sleep quality in patients undergoing abdominal hysterectomy. $J$ Clin Anesth. 2017;36:118-122. doi:10.1016/j.jclinane.2016.10.022;.

13. Su X, Meng ZT, Wu XH, et al. Dexmedetomidine for prevention of delirium in elderly patients after non-cardiac surgery: a randomised, double-blind, placebo-controlled trial. Lancet. 2016;388 (10054):1893-1902. doi:10.1016/s0140-6736(16)30580-3 
14. Li HJ, Li CJ, Wei XN, et al. Dexmedetomidine in combination with morphine improves postoperative analgesia and sleep quality in elderly patients after open abdominal surgery: a pilot randomized control trial. PLoS One. 2018;13(8):e0202008. doi:10.1371/journal. pone. 0202008

15. Cai J, Chen Y, Hao X, et al. Effect of Intraoperative dexmedetomidine dose on postoperative first night sleep quality in elderly surgery patients: a retrospective study with propensity score-matched analysis. Front Med (Lausanne). 2020;7:528. doi:10.3389/fmed.2020.00528;

16. Duan G, Wang K, Peng T, et al. The effects of intraoperative dexmedetomidine use and its different dose on postoperative sleep disturbance in patients who have undergone non-cardiac major surgery: a Real-World Cohort Study. Nat Sci Sleep. 2020;12:209-219. doi:10.2147/nss.S239706;

17. Shi H, Du X, Wu F, et al. Dexmedetomidine improves early postoperative neurocognitive disorder in elderly male patients undergoing thoracoscopic lobectomy. Exp Ther Med. 2020;20(4):3868-3877. doi:10.3892/etm.2020.9113

18. Liu X, Hu X, Li R, et al. Combination of post-fascia iliaca compartment block and dexmedetomidine in pain and inflammation control after total hip arthroplasty for elder patients: a randomized control study. J Orthop Surg Res. 2020;15(1):42. doi:10.1186/s13018-0201562-6

19. Sun Y, Jiang M, Ji Y, et al. Impact of postoperative dexmedetomidine infusion on incidence of delirium in elderly patients undergoing major elective noncardiac surgery: a randomized clinical trial. Drug Des Devel Ther. 2019;13:2911-2922. doi:10.2147/dddt.S208703;.

20. Mao Y, Sun X, Si L, et al. Perioperative dexmedetomidine fails to improve postoperative analgesic consumption and postoperative recovery in patients undergoing lateral thoracotomy for thoracic esophageal cancer: a Randomized, Double-Blind, Placebo-Controlled Trial. Pain Res Manag. 2020;2020:4145893. doi:10.1155/2020/4145893;.

21. Yu HY, Wang SY, Quan CX, et al. Dexmedetomidine alleviates postpartum depressive symptoms following cesarean section in Chinese women: a Randomized Placebo-Controlled Study. Pharmacotherapy. 2019;39(10):994-1004. doi:10.1002/phar.2320

22. Ghali AM, Shabana AM, El Btarny AM. The effect of low-dose dexmedetomidine as an adjuvant to levobupivacaine in patients undergoing vitreoretinal surgery under sub-tenon's block anesthesia. Anesth Analg. 2015;121(5):1378-1382. doi:10.1213/ane.00000 00000000908.

23. Shi C, Jin J, Pan Q, et al. Intraoperative use of dexmedetomidine promotes postoperative sleep and recovery following radical mastectomy under general anesthesia. Oncotarget. 2017;8(45):79397-79403. doi:10.18632/oncotarget.18157

24. Qin M, Chen K, Liu T, et al. Dexmedetomidine in combination with sufentanil for postoperative analgesia after partial laryngectomy. BMC Anesthesiol. 2017;17(1):66. doi:10.1186/s12871-017-0363-x

25. Jiang Z, Zhou G, Song Q, et al. Effect of intravenous oxycodone in combination with different doses of dexmedetomdine on sleep quality and visceral pain in patients after abdominal surgery: a Randomized Study. Clin J Pain. 2018;34(12):1126-1132. doi:10.1097/ ajp.0000000000000645

26. Tan WF, Guo B, Ma H, et al. Changes in postoperative night bispectral index of patients undergoing thoracic surgery with different types of anaesthesia management: a randomized controlled trial. Clin Exp Pharmacol Physiol. 2016;43(3):304-311. doi:10.1111/14401681.12530

27. Lu W, Fu Q, Luo X, et al. Effects of dexmedetomidine on sleep quality of patients after surgery without mechanical ventilation in ICU. Medicine (Baltimore). 2017;96(23):e7081. doi:10.1097/ md.0000000000007081
28. Krenk L, Jennum P, Kehlet H. Sleep disturbances after fast-track hip and knee arthroplasty. $B r \quad J$ Anaesth. 2012;109(5):769-775. doi:10.1093/bja/aes252.

29. Feng ZX, Dong H, Qu WM, et al. Oral delivered dexmedetomidine promotes and consolidates non-rapid eye movement sleep via sleep-wake regulation systems in mice. Front Pharmacol. 2018;9:1196. doi:10.3389/fphar.2018.01196;

30. Nelson LE, Lu J, Guo T, et al. The alpha2-adrenoceptor agonist dexmedetomidine converges on an endogenous sleep-promoting pathway to exert its sedative effects. Anesthesiology. 2003;98 (2):428-436. doi:10.1097/00000542-200302000-00024

31. Seidel WF, Maze M, Dement WC, et al. Alpha-2 adrenergic modulation of sleep: time-of-day-dependent pharmacodynamic profiles of dexmedetomidine and clonidine in the rat. $J$ Pharmacol Exp Ther. 1995;275(1):263-273.

32. Baker R, Gent TC, Yang Q, et al. Altered activity in the central medial thalamus precedes changes in the neocortex during transitions into both sleep and propofol anesthesia. J Neurosci. 2014;34 (40):13326-13335. doi:10.1523/jneurosci.1519-14.2014

33. Akeju O, Pavone KJ, Westover MB, et al. A comparison of propofoland dexmedetomidine-induced electroencephalogram dynamics using spectral and coherence analysis. Anesthesiology. 2014;121 (5):978-989. doi:10.1097/aln.0000000000000419

34. Oto J, Yamamoto K, Koike S, et al. Sleep quality of mechanically ventilated patients sedated with dexmedetomidine. Intensive Care Med. 2012;38(12):1982-1989. doi:10.1007/s00134-012-2685-y

35. Alexopoulou C, Kondili E, Diamantaki E, et al. Effects of dexmedetomidine on sleep quality in critically ill patients: a pilot study. Anesthesiology. 2014;121(4):801-807. doi:10.1097/ aln.0000000000000361

36. Chamadia S, Hobbs L, Marota S, et al. Oral dexmedetomidine promotes non-rapid eye movement stage 2 sleep in humans. Anesthesiology. 2020;133(6):1234-1243. doi:10.1097/aln.0000 000000003567

37. Akeju O, Hobbs LE, Gao L, et al. Dexmedetomidine promotes biomimetic non-rapid eye movement stage 3 sleep in humans: a pilot study. Clin Neurophysiol. 2018;129(1):69-78. doi:10.1016/j. clinph.2017.10.005

38. Deuschle M, Schredl M, Wisch C, et al. Serum brain-derived neurotrophic factor (BDNF) in sleep-disordered patients: relation to sleep stage N3 and rapid eye movement (REM) sleep across diagnostic entities. J Sleep Res. 2018;27(1):73-77. doi:10.1111/jsr.12577

39. Barends CR, Absalom A, van Minnen B, et al. Dexmedetomidine versus midazolam in procedural sedation. a systematic review of efficacy and safety. PLoS One. 2017;12(1):e0169525. doi:10.1371/ journal.pone.0169525

40. Constantin JM, Momon A, Mantz J, et al. Efficacy and safety of sedation with dexmedetomidine in critical care patients: a meta-analysis of randomized controlled trials. Anaesth Crit Care Pain Med. 2016;35(1):7-15. doi:10.1016/j.accpm.2015.06.012

41. Zhang X, Wang D, Shi M, et al. Efficacy and safety of dexmedetomidine as an adjuvant in epidural analgesia and anesthesia: a systematic Review and meta-analysis of Randomized Controlled Trials. Clin Drug Investig. 2017;37(4):343-354. doi:10.1007/s40261016-0477-9

42. Zeng Y, Wen Y, Yang J, et al. Comparing post-operative analgesic effects of varying doses of dexmedetomidine as an adjuvant to ropivacaine for ultrasound-guided dual transversus abdominis plane block following laparotomy for gynecologic malignancies. Exp Ther Med. 2020;20(2):860-867. doi:10.3892/etm.2020.8787 


\section{Publish your work in this journal}

Drug Design, Development and Therapy is an international, peerreviewed open-access journal that spans the spectrum of drug design and development through to clinical applications. Clinical outcomes, patient safety, and programs for the development and effective, safe, and sustained use of medicines are a feature of the journal, which has also been accepted for indexing on PubMed Central. The manuscript management system is completely online and includes a very quick and fair peer-review system, which is all easy to use. Visit http://www. dovepress.com/testimonials.php to read real quotes from published authors. 